\title{
Treatment of aggressive non-Hodgkin's lymphoma in frail patients: cardiac comorbidities and advanced age
}

\author{
Pieternella J Lugtenburg*,1, Alexander R Lyon ${ }^{2,3}$, Reinhard Marks ${ }^{4}$ \& Stefano Luminari ${ }^{5,6}$ \\ ${ }^{1}$ Department of Hematology, Erasmus MC Cancer Institute, Rotterdam, The Netherlands \\ ${ }^{2}$ Cardio-Oncology Service, Royal Brompton \& Harefield NHS Trust, London, UK \\ ${ }^{3}$ Imperial College London, National Heart \& Lung Institute, London, UK \\ ${ }^{4}$ Department of Hematology, Oncology \& Stem Cell Transplantation, Faculty of Medicine \& Medical Centre, University of Freiburg, \\ Freiburg, Germany \\ ${ }^{5}$ Hematology, Azienda USL IRCCS Reggio Emilia, Italy \\ ${ }^{6}$ CHIMOMO Department University of Modena \& Reggio Emilia, Reggio Emilia, Italy \\ *Author for correspondence: p.lugtenburg@erasmusmc.nl
}

The decisive factor in selecting a treatment regimen for a frail patient with aggressive non-Hodgkin's lymphoma is identifying whether a patient is fit enough to tolerate curative-intent anthracycline-containing regimens or too frail and therefore at risk of being undertreated. As cardiac comorbidities are an important contributor to both the health status and the selection of treatment, cardiovascular profiling and baseline risk stratification prior to treatment should be considered. Comprehensive geriatric assessment is an efficient means of identifying elderly patients with non-Hodgkin's lymphoma who may benefit from a curative treatment approach. If anthracycline-based therapy is not suitable, alternative treatment options are available in frail patients with cardiac comorbidities, but these must be adjusted to the patient's health status to achieve a maximal benefit-risk ratio.

\section{Keywords: cardiac comorbidities $\bullet$ elderly $\bullet$ frail $\bullet$ non-Hodgkin's lymphoma $\bullet$ treatment}

Diffuse large B-cell lymphoma is a potentially curable disease with currently available therapies. However, the treatments with the most potential for cure - the anthracycline-based chemotherapy regimens - are often a limited treatment option for the elderly, for the frail or for those with cardiac comorbidities. This is due to a higher susceptibility to treatment toxicity, a different physiology for elderly subjects and the role of comorbid conditions. Although there is a known disconnect between chronological age and the health status of a patient, there is currently a lack of assessment tools for physicians to accurately assess patient frailty.

Here we discuss the evaluation of frailty in older patients with aggressive non-Hodgkin's lymphoma (NHL) and we provide guidance on how to select treatment options depending on the health status of a patient. As cardiac comorbidities are an important contributor to both the health status and the selection of treatment, we especially focus on the diagnosis and monitoring of cardiac status in elderly patients with NHL.

Frail patients: what do we need to know?

NHL is one of the most common types of cancer, comprising 4.3\% of total cancers. Reports estimate 70,000 new cases and 20,000 deaths in the USA in 2017 [1,2]. Notably, NHL is most frequent in people aged 65-74 years [3], and as the population ages, NHL is expected to become more prevalent. Diffuse large B-cell lymphoma (DLBCL) is the most common form of NHL (31\% of cases) [4] and is curable in a large percentage of patients. The most widely accepted form of first-line therapy is R-CHOP (rituximab, cyclophosphamide, doxorubicin, vincristine, prednisone) [5].

Real-world data from the Netherlands show that trends in 5-year survival have improved over the last two decades. The prognosis for DLBCL has improved for all age groups, but less so for those over 75 years of age [6]. Consideration of the inferior outcome in the elderly is warranted; elderly patients may well be undertreated because

Future $\because \cdots$ Medicine 
prescribers are fearful of chemotherapy-induced toxicities. Alternatively, elderly patients are under-represented in clinical trials because they are usually not included due to multiple comorbidities or poor performance status. In their study, Van Spronsen et al. found that $66 \%$ of patients with NHL older than 60 years of age have one or more comorbidities [7]. Among these patients, cardiovascular disease is common, and has a strong prognostic influence. Overall 5-year survival in NHL patients (aged $>60$ years) was significantly less in those with two or more than in those with no comorbidities (hazard ratio: 1.9) [7]. Moreover, the more comorbidities a patient had, the less likely he or she was to receive curative therapy [7].

The primary reason for a poor outcome in elderly patients is their decreased ability to tolerate treatment [8]. Impaired bone-marrow function, altered drug metabolism and the presence of comorbid diseases can increase the number of treatment-related complications [9]. However, it is possible to improve outcomes for the elderly patient with NHL [9]. In a study of 150 elderly patients (median age: 83 years), treated with an R-mini-CHOP regimen, 2-year overall survival (OS) was 59\% (49-67\%), median progression-free survival was 21 months (95\% CI: 13 to upper limit not reached) and 2-year progression-free survival was 47\% (38-56\%). However, it should be noted that patients with severe comorbidities (especially cardiac comorbidities) were excluded from this study [9]. The decisive factor in selecting a treatment regimen for an elderly patient with aggressive NHL is to ascertain whether a patient is fit enough to tolerate curative-intent anthracycline-containing regimens or is too frail and therefore at risk of being undertreated. Definitions of frailty are changing and evolving but currently there is no consensus. Frailty is an important concept in cancer therapy because it is estimated that greater than half of older cancer patients have prefrailty or frailty, and these patients are at increased risk of chemotherapy intolerance, postoperative complications and mortality [10].

\section{Identifying \& monitoring cardiac comorbidity}

Considering that there is an increase in the number of long-surviving cancer patients who present with cardiovascular disease, there is growing importance for a multidisciplinary team approach with the inclusion of a cardiologist as part of a cancer patient's management team, and the development of new cardio-oncology specialists to meet this clinical need. This approach aims to prevent the hematology patient of today becoming the cardiac patient of tomorrow. The diametrically opposed relationship between cancer (cell division, increased cell number, angiogenesis, increased metabolic disease, drug/toxin resistance) and heart disease (failure of cell division/tissue repair, cell loss, ischemia, impaired/decreased energetic efficiency, increased sensitivity to toxins), that is, the cardio-oncology balance, provides healthcare providers with an opportunity to prevent, rather than respond to, therapeutic cardiotoxicity.

$\mathrm{R}-\mathrm{CHOP}$ remains the standard regimen in patients with aggressive NHL. However, the utility of doxorubicin is limited by cumulative, dose-related, progressive myocardial damage that can lead to congestive heart failure (CHF). Age is an important risk factor for doxorubicin-related CHF, with patients over 65 years of age experiencing a higher incidence of $\mathrm{CHF}$ when compared with patients younger than 65 years [11]. Retrospective analysis estimates the 5-year incidence of cardiac events in NHL patients treated with CHOP/R-CHOP to be approximately 29\% [12].

Anthracycline-based chemotherapy, which improves survival in aggressive NHL, is often withheld from elderly patients because of potentially cardiotoxic effects. In an American study that enrolled 9438 patients with DLBCL aged $\geq 65$ years, only $42 \%$ of patients received doxorubicin-based chemotherapy. Doxorubicin use was associated with a 29\% increase in CHF (95\% CI: 1.02-1.62), with absolute risk influenced by age, the number of doxorubicin cycles and the presence of pre-existing cardiovascular disease [13]. Real-world, prospective data from an Italian cohort ( $\mathrm{n}=2625$ patients receiving anthracycline-based chemotherapy) showed that the overall incidence of cardiotoxicity was $9 \%(n=226)$, the median time elapsed between end of chemotherapy treatment and the development of clinical cardiotoxicity was 3.5 months (Q1-Q3: 3-6) and that, in $98 \%$ of cases $(n=221)$, cardiotoxicity occurred within the first year [14]. It should be noted that the patients included in this real-world cohort were aged $>18$ years, that is, they were not an elderly population [14]. Such findings support a management strategy of assessing a patient's cardiac function within 3-4 months of initiating anthracycline-based chemotherapy.

The cardiac pathophysiology of anthracyclines is believed to involve the generation of reactive oxygen species, which results in damage to DNA, proteins and lipids, and leads to cellular dysfunction and cardiomyocyte death [15]. However, an alternative hypothesis suggests that the first step in anthracycline-mediated cardiomyocyte damage is independent of reactive oxygen species and depends instead on drug interactions with a type of topoisomerase, an enzyme that affects the tension and topologic features of DNA [16]. The elucidation of the underlying molecular mechanism(s) involved in anthracycline-induced injury to cardiac cells has resulted in the development of numerous novel, targeted therapies and an increasingly complex therapeutic milieu. 
Box 1. Baseline cardiovascular risk factors.

Previous cardiovascular disease

- Heart failure

- Ischemic heart disease

- Borderline left ventricular ejection fraction

- Left ventricular hypertrophy

- Elevated cardiac biomarkers

Demographic and other cardiovascular risk factors

- Age $>65$ years

- Female gender

- Hypertension

- Diabetes

- Renal failure

Previous cardiotoxic cancer treatment

- Anthracyclines

- Chest radiation with heart within radiation field

- VEGF TKIs

Lifestyle risk factors

- Obesity

- Smoking

TKI: Tyrosine kinase inhibitor.

Cardiologists advocate methods that can ameliorate the cardiotoxicity of doxorubicin; the most important is cardiovascular profiling and baseline risk stratification prior to treatment (Box 1) [19,21]. Other options include close surveillance of the cardiac status of patients with cardiac biomarkers/imaging during treatment, use of liposomal doxorubicin, dexrazoxane or the use of an alternative nonanthracycline-based chemotherapy [17-19]. There is no evidence that prolonged infusion ofanthracycline is more beneficial over bolus in terms of cardiotoxicity. Modern echocardiography techniques, such as speckle tracking echocardiography, permit early detection of cardiac dysfunction. Speckle tracking can be used to measure loss of cardiac global longitudinal strain, known to precede a reduction in ejection fraction and may be a useful predictor of when intervention is required to manage potential cardiotoxicity. Cardiac injury biomarker assessment is another useful tool during chemotherapy. Measurement of troponin I, after high cumulative dose $(300 \mathrm{mg})$ anthracycline chemotherapy, permits stratification of the risk of cardiac events in cancer patients in the 3 years thereafter [20].

Cardio-oncology services play a vital role in supporting patients experiencing cardiotoxicity during cancer treatment in order to complete their treatment safely [22]. The key to success is a multidisciplinary team approach across hematology, oncology and cardiology, all members of which work to minimize the delay to cancer treatment and support, not obstruct, cardiotoxic cancer treatments. Best practice includes rapid assessment without introducing delays, cardiac biomarker measurement (troponin, brain natriuretic peptide), resting echocardiography with speckle and $3 \mathrm{D}$ imaging, with stress echocardiography \pm contrast perfusion, cardiac MRI and computed tomography coronary angiography in selected patients, and same day results/treatment plan.

\section{Assessing the elderly or frail patient}

Clearly there is a need for individualized care in the elderly cancer patient. Yet, the definition for 'elderly' or 'frail' is not clear. Learned bodies, including the International Society of Geriatric Oncology, the European Organisation for Research and Treatment of Cancer, and the European Society for Medical Oncology (ESMO) suggest the term 'elderly' or 'old' should be used for all patients aged $>70$ years, yet, chronological age alone is a poor predictor of cancer treatment tolerance [23] and the heterogeneity of the older cancer patient requires a carefully tailored approach to care that considers individual frailty [10]. Due to the disconnect between numerical age and health status, prescribers face a dilemma when deciding who is suitable for treatment with a doxorubicin-containing regimen, and if suitable, which dose and therapeutic goals should be prescribed.

OS is but one of several treatment objectives in the elderly and/or frail patient with DLBCL. Consideration of the overall complexity of the patient is warranted, including tolerability, toxicity, comorbidities, potential for early death, quality of life, functional decline and, most importantly, the wishes of the patient [24-26]. Social status impacts on patient outcomes and geriatric assessment should also pay attention to the availability of family 
Table 1. European Society for Medical Oncology treatment guidelines for diffuse large B-cell lymphoma.

\begin{tabular}{|lll|}
\hline Fit, $60-80$ years & $>80$ years without cardiac dysfunction & Unfit or frail or $>60$ years with cardiac dysfunction \\
\hline R-CHOP & Attenuated regimens: R-mini-CHOP & Doxorubicin substitution \\
& & - Etoposide \\
& - Gemcitabine \\
& - Liposomal doxorubicin \\
& Others \\
\hline & R-CVP \\
R-bendamustine \\
\hline R-CHOP: Rituximab, cyclophosphamide, doxorubicin, vincristine, prednisone; R-CVP: Rituximab, cyclophosphamide, vincristine, prednisolone. \\
\hline
\end{tabular}

support, the living situation, social activity and caregiver burden [27]. Several well-validated tools are available for measuring comorbidities, such as, the Cumulative Illness Rating Scale (CIRS) and the Charlson Comorbidity Index (CCI) [28,29]. Recent data from Pardal et al. demonstrate that, simple prognostic models, like the CIRS, may aid in the selection of DLBCL patients who could benefit from a curative treatment [30].

According to International Society of Geriatric Oncology, a comprehensive geriatric assessment (CGA), with or without screening, and with follow-up, should be used in older cancer patients, to detect unaddressed problems, improve their functional status and possibly their survival [31]. Despite this recommendation, and the utility of the CGA, there are some disadvantages associated with this assessment. The CGA is relatively complex, timeconsuming and must be administered by a geriatrician. Simplified screening tools are needed and could include the G-8 geriatric screening tool [32], the Vulnerable Elders Survey (VES-13) [33] or the simplified CGA [34]. In 2015, Tucci et al. reported that the simplified CGA can identify elderly, nonfit patients with DLBCL, in whom curative treatment has no advantage over palliative care. Patients $(n=173)$ aged $>69$ years, treated with curative or palliative intent, were grouped according to CGA into fit, unfit and frail categories. The results showed that 2-year OS was significantly better in fit than in unfit patients ( $84 \mathrm{vs} 47 \%$; $\mathrm{p}<0.0001$ ) and that survival in unfit and frail patients was not significantly different. Curative treatment slightly improved 2-year OS in unfit (75 vs $45 \%$ ) but not in frail patients (44 vs 39\%). The authors suggest simplified CGA is an efficient means of identifying elderly patients with DLBCL who may benefit from a curative treatment approach [35].

\section{Therapy options for frail patients with DLBCL}

The complex pathophysiology of elderly patients with malignant lymphoma presents a clinical challenge. Less aggressive treatment may reduce the chances of cure, prolongation of life and/or symptom control, whereas an aggressive treatment may result in treatment-related morbidity and mortality, and/or compromised quality of life. Thus, a cornerstone in the decision-making process is to judge fitness for treatment [36]. The ESMO guidelines propose that 'fitness' is defined in the context of treatment feasibility with three different categories discriminating between 'fit', 'vulnerable' and 'terminally ill' patients. For an elderly 'fit' patient, it would be anticipated that application of the standard treatment, including more dose-intense approaches, would not be associated with a high risk of treatment-related or treatment-unrelated adverse events (AEs) compared with a young 'fit' patient. In contrast a 'vulnerable' patient would carry a high risk for treatment-related AEs or treatment-unrelated AEs when receiving standard treatment. Vulnerable patients would present a continuum, ranging from those who are just at the border of not being able to tolerate standard treatment to those who are close to being considered as terminally ill [36]. ESMO recommends that fit patients, aged 60-80 years, are offered curative therapy with R-CHOP (Table 1) [36,37]. The very elderly (aged $>80$ years), without cardiac dysfunction, should be treated with an attenuated anthracycline-containing regimen (R-mini-CHOP) and the elderly/frail, with cardiac dysfunction, are generally treated with an alternative or palliative therapy [36,37].

Liposomal doxorubicin combination therapy or the replacement of doxorubicin with etoposide is an option in elderly and cardiac frail patients. Evidence suggests that many patients (44-78\%) experience a complete response, but, at the expense of a decline in left ventricular ejection fraction [38-42]. One may also consider anthracycline-free combination therapy in elderly and cardiac frail patients, though this is known to result in an attenuation of the complete response [43]. 


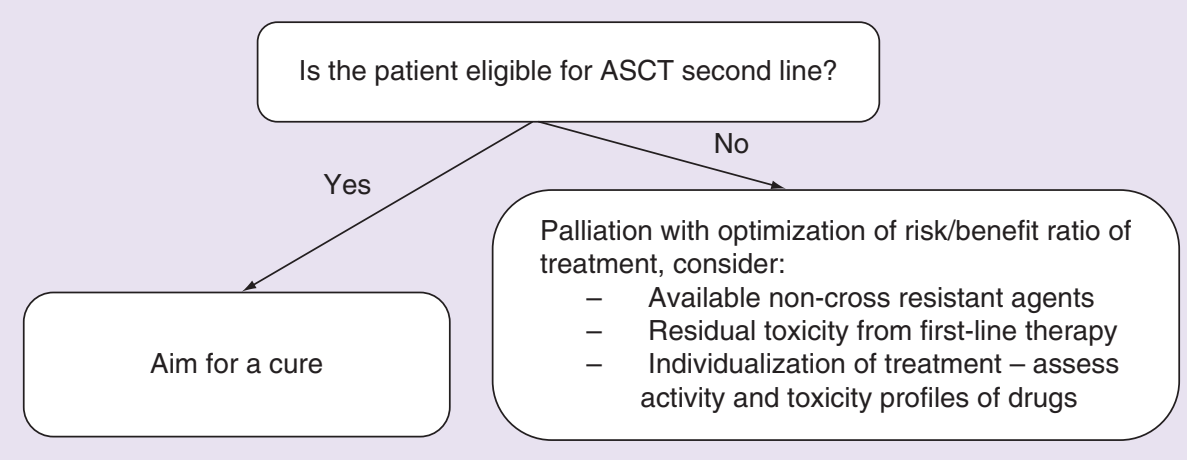

Figure 1. Proposed decisional algorithm in relapsed/refractory elderly, frail diffuse large B-cell lymphoma patients. ASCT: Autologous stem cell transplantation.

Box 2. Potential treatment regimens for elderly patients with relapsed/refractory diffuse large B-cell lymphoma not eligible for autologous stem cell transplantation.

- PECC: Prednisone, etoposide, chlorambucil, lomustine, $\pm \mathrm{R}$

- CEPP: Cyclophosphamide, etoposide, prednisone, procarbazine, $\pm \mathrm{R}$

- CEOP: Cyclophosphamide, etoposide, vincristine, prednisone, $\pm \mathrm{R}$

- GDP: Gemcitabine, dexamethasone, carboplatin, $\pm \mathrm{R}$

- GemOX: Gemcitabine, oxaliplatin, $\pm \mathrm{R}$

- Lenalidomide $\pm \mathrm{R}$

- Bendamustine $\pm \mathrm{R}$

- Palliative RT

R: Rituximab; RT: Radiation therapy.

Therapy options in patients with relapsed DLBCL after anthracycline-containing first-line therapy In the relapsed setting, cardiac toxicity is more difficult to manage compared with first-line therapy, as patients have often already received anthracycline first line or were treated with a doxorubicin-free regimen due to pre-existing cardiac problems. Figure 1 shows a suggested decisional algorithm in relapsed/refractory frail DLBCL patients. In addition, Box 2 lists some potential therapeutic strategies in elderly patients with relapsed/refractory DLBCL who are not eligible for high-dose chemotherapy followed by autologous stem cell transplantation [45]. Few randomized data are available from the literature to provide scientific support in the choice of a salvage therapy. Among available studies the use of pixantrone is supported by Phase III data. Data from a global Phase III study, in patients with aggressive NHL, who had relapsed after two or more previous chemotherapy regimens (including one standard anthracycline-containing protocol) demonstrated median progression-free survival of 5.2 months (95\% CI: 2.3 , 6.2) in patients treated with pixantrone monotherapy versus 2.6 months (95\% CI: 1.9, 3.5) in the comparator group [44]. The most common grade 3 or 4 adverse events in patients given pixantrone were uncomplicated, noncumulative neutropenia, leukopenia and thrombocytopenia; the severity of neutropenia did not increase with increasing cycle number. Notably, the cardiac events that were reported were predominantly asymptomatic grade 1 and 2 reductions in left ventricular ejection fraction, which did not increase with increasing pixantrone exposure. These results support the use of pixantrone as a treatment option for patients whose aggressive NHL has failed to respond to at least two previous chemotherapy regimens [44].

\section{Rituximab, cyclophosphamide, pixantrone, vincristine, prednisone}

Pixantrone was synthesized with the aim of reducing anthracycline-related cardiotoxicity, without compromising antitumor efficacy. In a Phase II study in which doxorubicin was substituted by pixantrone in untreated DLBCL patients, R-CPOP (rituximab, cyclophosphamide, pixantrone, vincristine, prednisone) demonstrated a modestly lower response rate than R-CHOP (rituximab, cyclophosphamide, doxorubicin, vincristine, prednisone) but similar progression-free and event-free survivals and a lower incidence of severe cardiac events [46]. These data do not support 
the use of R-CPOP as first-line therapy but demonstrate a substantial difference in the cardiotoxicity of pixantrone compared with doxorubicin when used as first-line therapy [46].

\section{Conclusion}

The incidence of cardiotoxicity is increasing among patients undergoing anthracycline-based chemotherapy, particularly in the frail population where a careful assessment of the benefit-risk ratio is mandatory. Performing a baseline cardiovascular assessment prior to the instigation of treatment is a vital part of cardio-oncology patient-centered care. Easy-to-use, validated tools for assessing elderly patients are available, and can guide treatment goals and options. Once the patient status has been determined, alternative treatment options are available in frail patients with cardiac comorbidities, but these must be adjusted to the patient's health status to achieve a maximal benefitrisk ratio. In conclusion, frail patients represent an unmet need in lymphoma, and well-designed clinical trials are warranted.

\section{Future perspective}

The treatment of elderly patients with aggressive NHL is often complicated due to comorbidities and they are often on many drugs with potential interactions. In the future, our hope is that frail patients will receive tailored treatment, which is equivalent to that prescribed to younger patients but which takes into consideration the socioeconomic issues and wishes of the elderly patient. Key factors in achieving this goal will be a universally accepted definition of frailty, routine geriatric and baseline cardiovascular risk assessment, and the development of new drug entities with minimal or no cardiotoxicity, that can be administered at doses that do not compromise antitumor efficacy.

The literature is awash with studies looking at different frailty scaling and rating systems, which aim to assess the fitness of older patients, particularly their ability to tolerate cancer treatments. None of these rating scales are perfect, and all have their champions and detractors. In the future, we predict that, a simple, reliable geriatric assessment tool will be available to enable professionals to treat and manage older people, and this assessment will be used to guide therapeutic choices.

Treatment decisions are often based on clinical judgement, which varies between clinicians and may also be subject to bias. Our ambition is that, by 2030, patients will be managed by multidisciplinary geriatric oncology services, including geriatricians, hematologists, oncologists and cardiologists, all of whom will be working to minimize the delay to treatment and to support, not obstruct, cancer treatments.

As things stand, we believe that our healthcare systems are not adequately prepared to meet the challenges of an aging population with cancer. Clearly, more research is needed to address the specific needs of the elderly. A range of national policies and practices is being introduced to help address this gap, but more must be done to ensure that cancer services are equipped to meet the demands of the future, fronted by a global cancer care strategy for the frail patient.

\section{Contributors}

This article is based on an EHA Educational Session entitled Treatment of Aggressive NHL in Frail Patients: Cardiac Comorbidities and Advanced Age at the European Haematology Association Annual Congress in Stockholm, 16 June 2018. The manuscript was developed based on presentations made by the authors during that session. All authors reviewed and revised the manuscript, and agreed on the final version.

Financial \& competing interests disclosure

PJ Lugtenburg received research funding from Servier, Takeda and Roche; advisory board honoraria from Servier, Takeda, Roche, Janssen-Cilag, Merck, Bristol-Myers Squibb and Celgene. AR Lyon has received speaker, advisory board or consultancy fees and/or research grants from Servier, Pfizer, Novartis, Amgen, Clinigen Group, Takeda, Roche, Eli Lily, Eisai, Bristol-Myers Squibb, Ferring Pharmaceuticals and Boehringer Ingelheim. R Marks has received speaker and advisory honoraria from Servier, Roche and Merck. $S$ Luminari has received honoraria from Servier. The authors have no other relevant affiliations or financial involvement with any organization or entity with a financial interest in or financial conflict with the subject matter or materials discussed in the manuscript apart from those disclosed.

Third party medical writing support was supplied by L McMurdo, Method, UK, funded by Servier Medical Affairs, Suresnes, France. 


\section{Open access}

This work is licensed under the Attribution-NonCommercial-NoDerivatives 4.0 Unported License. To view a copy of this license, visit http://creativecommons.org/licenses/by-nc-nd/4.0

\section{Executive summary}

Frail patients: what do we need to know?

- There is a known disconnect between chronological age and the health status of a patient.

- The decisive factor in selecting a treatment regimen for a frail patient with aggressive non-Hodgkin's lymphoma is to ascertain whether a patient is fit enough to tolerate curative-intent anthracycline-containing regimens or too frail and therefore at risk of being undertreated.

Identifying \& monitoring cardiac comorbidity

- The utility of doxorubicin is limited by cumulative, dose-related, progressive myocardial damage that can lead to congestive heart failure.

- Cardiologists advocate methods that can ameliorate the cardiotoxicity of doxorubicin; the most important is cardiovascular profiling and baseline risk stratification prior to treatment.

- Cardio-oncology services play a vital role in supporting patients experiencing cardiotoxicity during cancer treatment to complete their treatment safely.

Assessing the elderly or frail patient

- Consideration of the overall complexity of the patient is warranted, including tolerability, toxicity, comorbidities, potential for early death, quality of life, functional decline, social situation and most importantly, the wishes of the patient.

- Comprehensive geriatric assessment, with or without screening, and with follow-up, should be used in older cancer patients, to detect unaddressed problems, improve their functional status and possibly their survival.

Treatment options for frail patients

- Alternative treatment options are available in frail patients with cardiac comorbidities, but these must be adjusted to the patient's health status to achieve a maximal benefit-risk ratio.

- The European Society for Medical Oncology (ESMO) recommends that fit patients, aged 60-80 years, are offered curative therapy with R-CHOP; very elderly patients (aged $>80$ years), without cardiac dysfunction, should be treated with an attenuated anthracycline-containing regimen (R-mini-CHOP); and the elderly/frail, with cardiac dysfunction should be treated with an alternative or palliative therapy.

- In the relapsed setting, cardiac toxicity is more difficult to manage than in first-line therapy, as patients have often already received anthracycline first line or were treated with a doxorubicin-free regimen due to pre-existing cardiac problems.

- Few randomized studies are available from the literature to provide scientific support in the choice of a salvage therapy. Among available studies the use of pixantrone in the multiply relapsed/refractory setting is supported by Phase III data.

\section{References}

1. Sant M, Minicozzi P, Mounier M et al. Survival for haematological malignancies in Europe between 1997 and 2008 by region and age: results of EUROCARE-5, a population-based study. Lancet Oncol. 15(9), 931-942 (2014).

2. Siegel RL, Miller KD, Jemal A. Cancer statistics, 2017. CA Cancer J. Clin. 67(1), 7-30 (2017).

3. National Cancer Institute. Cancer stat facts: non-Hodgkin lymphoma. (2017).

4. Armitage JO, Weisenburger DD. New approach to classifying non-Hodgkin's lymphomas: clinical features of the major histologic subtypes. Non-Hodgkin’s Lymphoma Classification Project. J. Clin. Oncol. 16(8), 2780-2795 (1998).

5. Sehn LH, Gascoyne RD. Diffuse large B-cell lymphoma: optimizing outcome in the context of clinical and biologic heterogeneity. Blood 125(1), 22-32 (2015).

6. Issa DE, Van De Schans SA, Chamuleau ME et al. Trends in incidence, treatment and survival of aggressive B-cell lymphoma in the Netherlands 1989-2010. Haematologica 100(4), 525-533 (2015).

7. Van Spronsen DJ, Janssen-Heijnen ML, Lemmens VE, Peters WG, Coebergh JW. Independent prognostic effect of co-morbidity in lymphoma patients: results of the population-based Eindhoven Cancer Registry. Eur. J. Cancer 41(7), 1051-1057 (2005).

8. Armitage JO, Potter JF. Aggressive chemotherapy for diffuse histiocytic lymphoma in the elderly: increased complications with advancing age. J. Am. Geriatr. Soc. 32(4), 269-273 (1984).

9. Peyrade F, Jardin F, Thieblemont $\mathrm{C}$ et al. Attenuated immunochemotherapy regimen (R-miniCHOP) in elderly patients older than 80 years with diffuse large B-cell lymphoma: a multicentre, single-arm, Phase II trial. Lancet Oncol. 12(5), 460-468 (2011).

10. Handforth C, Clegg A, Young C et al. The prevalence and outcomes of frailty in older cancer patients: a systematic review. Ann. Oncol. 26(6), 1091-1101 (2015). 
11. Swain SM, Whaley FS, Ewer MS. Congestive heart failure in patients treated with doxorubicin: a retrospective analysis of three trials. Cancer 97(11), 2869-2879 (2003).

12. Limat S, Daguindau E, Cahn JY et al. Incidence and risk-factors of CHOP/R-CHOP-related cardiotoxicity in patients with aggressive non-Hodgkin's lymphoma. J. Clin. Pharm. Ther. 39(2), 168-174 (2014).

13. Hershman DL, McBride RB, Eisenberger A, Tsai WY, Grann VR, Jacobson JS. Doxorubicin, cardiac risk factors, and cardiac toxicity in elderly patients with diffuse B-cell non-Hodgkin's lymphoma. J. Clin. Oncol. 26(19), 3159-3165 (2008).

14. Cardinale D, Colombo A, Bacchiani G et al. Early detection of anthracycline cardiotoxicity and improvement with heart failure therapy. Circulation 131(22), 1981-1988 (2015).

15. Sawyer DB. Anthracyclines and heart failure. N. Engl. J. Med. 368(12), 1154-1156 (2013).

16. Zhang S, Liu X, Bawa-Khalfe T et al. Identification of the molecular basis of doxorubicin-induced cardiotoxicity. Nat. Med. 18(11), 1639-1642 (2012).

17. Ameri P, Canepa M, Anker MS et al. Cancer diagnosis in patients with heart failure: epidemiology, clinical implications and gaps in knowledge. Eur. J. Heart Fail. 20(5), 879-887 (2018).

18. Armenian SH, Lacchetti C, Barac A et al. Prevention and monitoring of cardiac dysfunction in survivors of adult cancers: American Society of Clinical Oncology Clinical Practice Guideline. J. Clin. Oncol. 35(8), 893-911 (2017).

19. Zamorano JL, Lancellotti P, Rodriguez Munoz D et al. 2016 ESC Position Paper on cancer treatments and cardiovascular toxicity developed under the auspices of the ESC Committee for Practice Guidelines: the task force for cancer treatments and cardiovascular toxicity of the European Society of Cardiology (ESC). Eur. Heart. J. 37(36), 2768-2801 (2016).

20. Cardinale D, Sandri MT, Colombo A et al. Prognostic value of troponin I in cardiac risk stratification of cancer patients undergoing high-dose chemotherapy. Circulation 109(22), 2749-2754 (2004).

21. Herrmann J, Lerman A, Sandhu NP, Villarraga HR, Mulvagh SL, Kohli M. Evaluation and management of patients with heart disease and cancer: cardio-oncology. Mayo Clin. Proc. 89(9), 1287-1306 (2014).

22. Lancellotti P, Suter TM, Lopez-Fernandez T et al. Cardio-oncology services: rationale, organization, and implementation: a report from the ESC Cardio-Oncology council. Eur. Heart J. doi:10.1093/eurheartj/ehy453 (2018) (Epub ahead of print).

23. Quoix E, Zalcman G, Oster JP et al. Carboplatin and weekly paclitaxel doublet chemotherapy compared with monotherapy in elderly patients with advanced non-small-cell lung cancer: IFCT-0501 randomised, Phase III trial. Lancet 378(9796), 1079-1088 (2011).

24. Soubeyran P, Fonck M, Blanc-Bisson C et al. Predictors of early death risk in older patients treated with first-line chemotherapy for cancer. J. Clin. Oncol. 30(15), 1829-1834 (2012).

25. Luminari S, Cesaretti M, Rashid I et al. Incidence, clinical characteristics and survival of malignant lymphomas: a population-based study from a cancer registry in northern Italy. Hematol. Oncol. 25(4), 189-197 (2007).

26. Hoppe $\mathrm{S}$, Rainfray M, Fonck $\mathrm{M}$ et al. Functional decline in older patients with cancer receiving first-line chemotherapy. J. Clin. Oncol. 31(31), 3877-3882 (2013).

27. Wildiers H, Heeren P, Puts M et al. International Society of Geriatric Oncology consensus on geriatric assessment in older patients with cancer. J. Clin. Oncol. 32(24), 2595-2603 (2014).

28. Extermann M, Overcash J, Lyman GH, Parr J, Balducci L. Comorbidity and functional status are independent in older cancer patients. J. Clin. Oncol. 16(4), 1582-1587 (1998).

29. Charlson ME, Pompei P, Ales KL, MacKenzie CR. A new method of classifying prognostic comorbidity in longitudinal studies: development and validation. J. Chronic Dis. 40(5), 373-383 (1987).

30. Pardal E, Diez Baeza E, Salas Q et al. A new prognostic model identifies patients aged 80 years and older with diffuse large B-cell lymphoma who may benefit from curative treatment: a multicenter, retrospective analysis by the Spanish GELTAMO group. Am. J. Hematol. 93(7), 867-873 (2018).

31. Extermann M, Aapro M, Bernabei R et al. Use of comprehensive geriatric assessment in older cancer patients: recommendations from the task force on CGA of the International Society of Geriatric Oncology (SIOG). Crit. Rev. Oncol. Hematol. 55(3), 241-252 (2005).

32. Bellera CA, Rainfray M, Mathoulin-Pelissier $S$ et al. Screening older cancer patients: first evaluation of the G-8 geriatric screening tool. Ann. Oncol. 23(8), 2166-2172 (2012).

33. Saliba D, Elliott M, Rubenstein LZ et al. The Vulnerable Elders Survey: a tool for identifying vulnerable older people in the community. J. Am. Geriatr. Soc. 49(12), 1691-1699 (2001).

34. Spina M, Balzarotti M, Uziel L et al. Modulated chemotherapy according to modified comprehensive geriatric assessment in 100 consecutive elderly patients with diffuse large B-cell lymphoma. Oncologist 17(6), 838-846 (2012).

35. Tucci A, Martelli M, Rigacci $\mathrm{L}$ et al. Comprehensive geriatric assessment is an essential tool to support treatment decisions in elderly patients with diffuse large B-cell lymphoma: a prospective multicenter evaluation in 173 patients by the Lymphoma Italian Foundation (FIL). Leuk. Lymphoma 56(4), 921-926 (2015).

36. Buske $\mathrm{C}$, Hutchings $\mathrm{M}$, Ladetto $\mathrm{M}$ et al. ESMO Consensus Conference on malignant lymphoma: general perspectives and recommendations for the clinical management of the elderly patient with malignant lymphoma. Ann. Oncol. 29(3), 544-562 (2018). 
37. Tilly H, Gomes Da Silva M, Vitolo U et al. Diffuse large B-cell lymphoma (DLBCL): ESMO Clinical Practice Guidelines for diagnosis, treatment and follow-up. Ann. Oncol. 26(Suppl. 5), v116-v125 (2015).

38. Luminari S, Viel E, Ferreri AJM et al. Nonpegylated liposomal doxorubicin combination regimen in patients with diffuse large B-cell lymphoma and cardiac comorbidity. Results of the HEART01 Phase II trial conducted by the Fondazione Italiana Linfomi. Hematol. Oncol. 36(1), 68-75 (2018).

39. Rohlfing S, Aurich M, Schoning T, Ho AD, Witzens-Harig M. Nonpegylated liposomal doxorubicin as a component of R-CHOP is an effective and safe alternative to conventional doxorubicin in the treatment of patients with diffuse large B-cell lymphoma and preexisting cardiac diseases. Clin. Lymphoma Myeloma Leuk. 15(8), 458-463 (2015).

40. Oki Y, Ewer MS, Lenihan DJ et al. Pegylated liposomal doxorubicin replacing conventional doxorubicin in standard R-CHOP chemotherapy for elderly patients with diffuse large B-cell lymphoma: an open label, single arm, Phase II trial. Clin. Lymphoma Myeloma Leuk. 15(3), 152-158 (2015).

41. Corazzelli G, Frigeri F, Arcamone M et al. Biweekly rituximab, cyclophosphamide, vincristine, non-pegylated liposome-encapsulated doxorubicin and prednisone (R-COMP-14) in elderly patients with poor-risk diffuse large B-cell lymphoma and moderate to high 'life threat' impact cardiopathy. Br. J. Haematol. 154(5), 579-589 (2011).

42. Gimeno E, Sanchez-Gonzalez B, Alvarez-Larran A et al. Intermediate dose of nonpegylated liposomal doxorubicin combination (R-CMyOP) as first line chemotherapy for frail elderly patients with aggressive lymphoma. Leuk. Res. 35(3), 358-362 (2011).

43. Fields PA, Townsend W, Webb A et al. De novo treatment of diffuse large B-cell lymphoma with rituximab, cyclophosphamide, vincristine, gemcitabine, and prednisolone in patients with cardiac comorbidity: a United Kingdom National Cancer Research Institute trial. J. Clin. Oncol. 32(4), 282-287 (2014).

44. Pettengell R, Coiffier B, Narayanan G et al. Pixantrone dimaleate versus other chemotherapeutic agents as a single-agent salvage treatment in patients with relapsed or refractory aggressive non-Hodgkin lymphoma: a Phase III, multicentre, open-label, randomised trial. Lancet Oncol. 13(7), 696-706 (2012).

45. Gisselbrecht C. Use of rituximab in diffuse large B-cell lymphoma in the salvage setting. Br. J. Haematol. 143(5), 607-621 (2008).

46. Herbrecht R, Cernohous P, Engert A et al. Comparison of pixantrone-based regimen (CPOP-R) with doxorubicin-based therapy (CHOP-R) for treatment of diffuse large B-cell lymphoma. Ann. Oncol. 24(10), 2618-2623 (2013). 
